\section{Über den Bau der Gerüste in thixotropen Gelen}

Von Armin Weiss und R. Frank

Eduard-Zintl-Institut für anorganische und physikalische Chemie der Technischen Hochschule Darmstadt

(Z. Naturforschg. 16 b, 141-142 [1961] ; eingeg. am 15. Oktober 1960)

Aus Untersuchungen an isochoren Kaolinitgelen mit austauschfähigen Calciumionen in Wasser bzw. nach $\mathrm{Zu}$ satz bestimmter Mengen von $\mathrm{Na}_{2} \mathrm{CO}_{3}$ (300 mval/100 g Kaolinit), $\mathrm{NaF}$ (200 mval/100 g Kaolinit) oder $\mathrm{K}_{2} \mathrm{CO}_{3}$ (73 mval/100 g Kaolinit) zur Ausfällung der Calciumionen geht hervor, daß die Gerüste in thixotrop erstarrten Gelen bei ein und derselben festen Phase je nach der Art der im System vorhandenen gelösten und dissoziationsfähigen Ionen recht unterschiedlich gebaut sein können.

Beim Zentrifugieren dieser Gele mit gleichem thixotropem Grenzvolumen ${ }^{1}$ erniedrigt sich ihr Volumen unter Ausbildung einer scharfen Grenzfläche gegen das überstehende Wasser recht verschieden, entsprechend einer unterschiedlichen Festigkeit der Gerüste. Nach $\mathrm{Zu}$ satz der Elektrolyte ist die Volumenverringerung unter sonst gleichen Versuchsbedingungen wesentlich stärker als bei dem Gel in dest. Wasser (Tab. 1). Darüber hinaus ist mit letzterem die Volumenänderung beim Stehen teilweise reversibel, während sie mit den elektrolythaltigen Gelen irreversibel bleibt.

Dieser Befund läßt sich am einfachsten deuten mit der Annahme, daß in dem „elastischen" thixotropen Gel ein Bändergerüst (Abb. 1) mit großer Festigkeit, im un-

1 Flüssigkeitsgehalt/g Kaolinit bei 6 sec Erstarrungszeit, vgl. hierzu W. Czerch, Dissertation, Darmstadt 1958; W. Czerch,

K. Frühauf u. U. Hofmann, Ber. dtsch. keram. Ges. 37, 255 [1960].

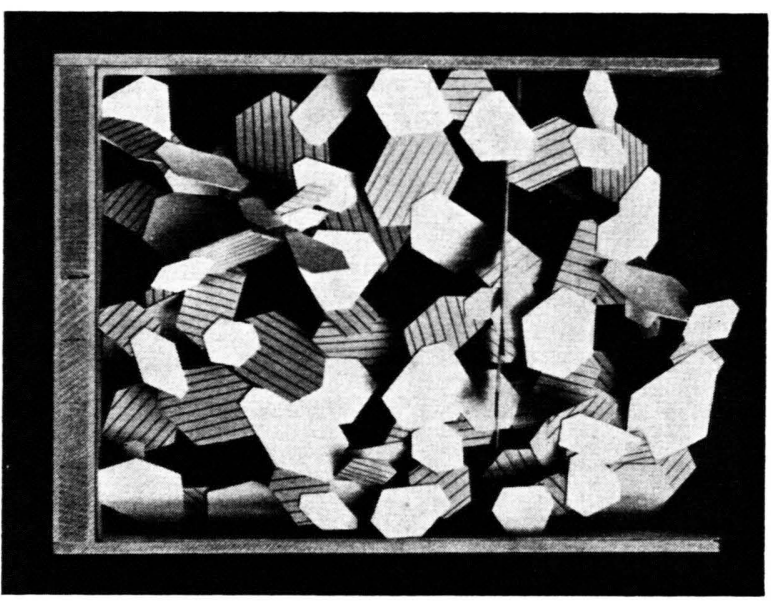

Abb. 1. Bändergerüst in den thixotrop erstarrten Gelen von Ca-Kaolinit in dest. Wasser.

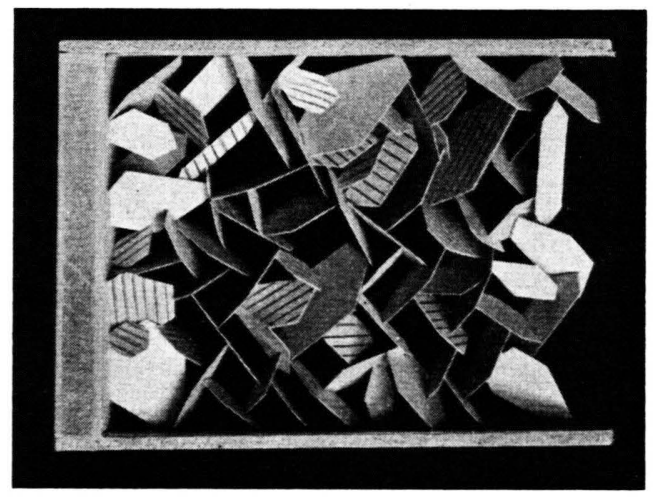

Abb. 2. Kartenhausgerüst in den thixotrop erstarrten Gelen von Ca-Kaolinit in Wasser nach Zusatz von $\mathrm{Na}_{2} \mathrm{CO}_{3}, \mathrm{NaF}$ oder $\mathrm{K}_{2} \mathrm{CO}_{3}$.

\begin{tabular}{|c|c|c|c|c|c|c|c|c|}
\hline \multirow[b]{3}{*}{$g \cdot t$} & \multicolumn{8}{|c|}{ Volumen bzw. Volumenänderung der thixotropen Gele } \\
\hline & \multicolumn{2}{|c|}{$\begin{array}{l}\text { Ca-Kaolinit in dest. } \\
\qquad \mathrm{H}_{2} \mathrm{O}\end{array}$} & \multicolumn{2}{|c|}{$\begin{array}{c}\text { Ca-Kaolinit in } \mathrm{H}_{2} \mathrm{O} \\
+300 \text { mval } \mathrm{Na}_{2} \mathrm{CO}_{3} / \\
100 \mathrm{~g} \text { Kaolinit }\end{array}$} & \multicolumn{2}{|c|}{$\begin{array}{c}\text { Ca-Kaolinit in } \mathrm{H}_{2} \mathrm{O} \\
+200 \mathrm{mval} \mathrm{NaF} / \\
100 \mathrm{~g} \mathrm{Kaolinit}\end{array}$} & \multicolumn{2}{|c|}{$\begin{array}{c}\text { Ca-Kaolinit in } \mathrm{H}_{2} \mathrm{O} \\
+73 \text { mval } \mathrm{K}_{2} \mathrm{CO}_{3} / \\
100 \text { g Kaolinit }\end{array}$} \\
\hline & $\begin{array}{c}\text { sofort nach } \\
\text { dem Zen- } \\
\text { trifugieren }\end{array}$ & $\begin{array}{c}\text { Volumen- } \\
\text { änderung } \\
\text { nach } \\
30 \text { Tagen } \\
\text { Standzeit } \\
{[\%]}\end{array}$ & $\begin{array}{c}\text { sofort nach } \\
\text { dem Zen- } \\
\text { trifugieren } \\
\\
{[\mathrm{ml}]}\end{array}$ & $\begin{array}{c}\text { Volumen- } \\
\text { änderung } \\
\text { nach } \\
\text { 30 Tagen } \\
\text { Standzeit } \\
{[\%]}\end{array}$ & $\begin{array}{l}\text { sofort nach } \\
\text { dem Zen- } \\
\text { trifugieren }\end{array}$ & $\begin{array}{c}\text { Volumen- } \\
\text { änderung } \\
\text { nach } \\
30 \text { Tagen } \\
\text { Standzeit } \\
{[\%]}\end{array}$ & $\begin{array}{c}\text { sofort nach } \\
\text { dem Zen- } \\
\text { trifugieren }\end{array}$ & $\begin{array}{c}\text { Volumen- } \\
\text { änderung } \\
\text { nach } \\
\text { 30 Tagen } \\
\text { Standzeit } \\
{[\%]}\end{array}$ \\
\hline 0 & 5,3 & $-8,3$ & 5,3 & 0 & 5,3 & 0 & 5,3 & 0 \\
\hline $0,5 \cdot 10^{4}$ & 5,3 & $-8,3$ & 5,2 & 0 & 4,9 & 0 & 4,6 & 0 \\
\hline $1,0 \cdot 10^{4}$ & 5,3 & $-8,3$ & 5,0 & 0 & 4,7 & 0 & $\begin{array}{l}\mathbf{T}, 0 \\
4,3\end{array}$ & 0 \\
\hline $0,2 \cdot 10^{5}$ & 5,1 & $\begin{array}{l}-4,3 \\
-\end{array}$ & 4,5 & 0 & 4,4 & 0 & 4,0 & 0 \\
\hline $0,2 \cdot 10^{6}$ & 2,6 & $+16,5$ & 2,2 & 0 & 2,3 & 0 & 2,4 & 0 \\
\hline $0,5_{5} \cdot 10^{6}$ & 2,2 & $+22,7$ & 2,0 & 0 & 1,8 & $+5,9$ & 2,0 & $+2,4$ \\
\hline
\end{tabular}

Tab. 1. Verhalten thixotroper Kaolinitproben mit gleichem Grenzvolumen und gleicher Erstarrungszeit beim Zentrifugieren. (Thixotropes Grenzvolumen $\mathrm{ml} / 3 \mathrm{~g}$ Kaolinit; Erstarrungszeit $6 \mathrm{sec}$ ). 
elastischen Gel dagegen ein lockeres Kartenhausgerüst ${ }^{2}$ (Abb. 2) vorliegt. Die nur teilweise Reversibilität der Volumenänderung im Bändergerüst hängt damit zusammen, daß nur die mit der Plättchenebene senkrecht zum Schwerefeld liegenden Bänder eine weitgehend elastische Verformung erleiden.

Mit diesen Vorstellungen über den unterschiedlichen Bau der Gerüste stimmt überein, daß bei Kaolinit die Kristalle wegen der dichten Packung der austauschfähigen Kationen nur durch zweiwertige Kationen zu festen Bändern vernetzt werden können, aber nicht durch einwertige Kationen ${ }^{3}$.

Bei den Werten in der Tab. 1 fällt auf, daß das Volumen des Bändergerüstes von reinem Ca-Kaolinit in dest. Wasser bei der kurzzeitigen Einwirkung eines verhältnismäßig starken Schwerefeldes $(200 \mathrm{~g}) \mathrm{kaum}$, unter dem Einfluß des Erdfeldes ( $1 \mathrm{~g}$ ) bei längerem Stehen dagegen wesentlich erniedrigt wird. Dieses Verhalten erinnert an die „dilatancy“ genannte Erscheinung ${ }^{4}$

2 U. Hofmann, Chemie 55, 283 [1942].

3 A. Werss, Z. anorg. allg. Chem. 299, 92 [1959].
(O s b orn e-R e y nolds-Phänomen). Es hängt wahrscheinlich damit zusammen, daß bei einer momentanen Volumverkleinerung durch Veränderung der Bänder etwa nach

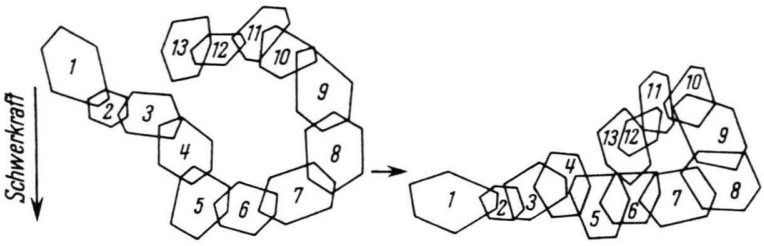

alle in der Oberfläche fixierten negativen Ladungen praktisch gleichzeitig gegen die austauschfähigen $\mathrm{Ca}$ Ionen verschoben werden müßten. Eine solche gleichzeitige Verschiebung ist aber unwahrscheinlich, wie der zeitliche Verlauf des Kationen-Austausches zeigt.

Eine ausführliche Beschreibung der Versuche erfolgt an anderer Stelle.

4 Osborne Reynolds, Philos. Mag. (5) 20, 469 [1885]; H. Freundlich, Thixotropy, Paris 1935; E. J. W. Verwey u. J. H. de Boer, Recueil Trav. chim. Pays-Bas 57, 383 [1938].
Aminosäuren. 5. Mitt. ${ }^{1}$ :

\section{Altersabhängigkeit des Gehaltes einiger Aminosäuren im Blut und "Normalisierung“" durch Cysteinzufuhr}

\section{Von Randolph Riemschneider}

Aus der Freien Universität Berlin, Lehrstuhl für Biochemie, und dem Hamburger Privatlaboratorium des Verfassers ${ }^{2}$

(Z. Naturforschg. 16 b, 142-143 [1961] ; eingeg. am 19. August 1960)

Vom Alter tierischer Organismen abhängige Konzentrations-Änderungen von Aminosäuren im Blut sind mehrfach beobachtet worden. Zum Beispiel sinkt nach C. I. Parhon und S. Oeriu ${ }^{3}$ bei Ratten mit zunehmendem Alter die Methionin-Konzentration des Blutserums, während die Cystin-Konzentration ansteigt. Cysteingaben bewirken Konzentrations-Änderungen in dem Sinne, daß Methionin- bzw. Cystinspiegel auf Werte erhöht bzw. gesenkt werden, die man bei jungen Ratten findet. Die Untersuchungen von W. C. Rose und R. L. $\mathrm{W}_{\text {Ixом }}{ }^{4}$ am Menschen weisen ebenfalls in diese Richtung. Diese Beobachtungen interessierten uns sowohl im Rahmen von Untersuchungen über die „Schutzwirkungen" von Verbindungen mit aktiven und poten tiellen SH-Gruppen und Vorstufen davon ${ }^{5}$ sowie auch im Zusammenhang mit Versuchen zur Beeinflussung von Stoffwechselvorgängen durch unphysiologische Aminosäuren, die der Asparaginsäure und Glutamin-

1 4. Mitt., Mh. Chem. 90, 924 [1959].

2 Anschrift für den Schriftverkehr: Hamburg-Blankenese, Postfach 13664.

3 C. I. Parhon u. S. Oeriv, 4th Congr. internat. Assoc. Gerontology, Meran, Juli 1957. säure konstitutionell nahestehen [d.h. zur Auslösung von Prozessen, die Störungen des Eiweißhaushaltes und der Fortpflanzungsfähigkeit von Schädlingen (Insekten) zum Ziel haben $]^{6}$.

In diesem Zusammenhang und zur weiteren Stütze einer „Altersabhängigkeit des Gleichgewichtes $\mathrm{R}-\mathrm{SS}-\mathrm{R} \leftrightarrows 2 \mathrm{R}-\mathrm{SH}^{\text {“ }}$ haben wir an einem größeren Tiermaterial zunächst folgende Teilfragen studiert: Ändern sich Asparagin- und Glutaminsäurespiegel mit zunehmendem Alter der Versuchstiere und wenn ja, vermag Cysteinzufuhr „Normalisierung“ zu bewirken? Läßt sich ein Einfluß von Cysteinvorstufen, z. B. Serin, oder auch von Verbindungen, die bei der Spaltung im Organismus Cystein liefern, z. B. Thiazolidincarbonsäure, auf das Cystein-Cystin-Gleichgewicht nachweisen?

$$
\text { Versuchsergebnisse }
$$

Die an 910 Versuchstieren (Ratten und Meerschweinchen) gewonnenen Resultate sind in Tab. 1 zusammengestellt. Man erkennt, daß der Asparaginsäuregehalt des Blutserums der Tiere mit zunehmendem Alter sinkt, der Glutaminsäurespiegel erhöht wird (Versuchsreihe Nr. 1-3, 6-8). Durch perorale Cysteinapplikation an alte Versuchstiere läßt sich „Normalisierung“ der Asparagin- und Glutaminsäure-Konzentrationen erreichen (Nr. 4 und 9). - Serinzufuhr bewirkt bei Tieren vorgeschrittenen Alters Erhöhung des Cystein- und Absin-

4 W. C. Rose u. R. L. Wrxom, J. biol. Chemistry 216, 764 [1955].

5 Vgl. z. B. R. Riemschneider, Z. Naturforschg. 16 b, 75 [1961].

${ }^{6}$ Unveröffentlichte Versuche. 